\title{
Tunable optical lattices in the near-field of a few-mode nanophotonic waveguide
}

\author{
Christophe Pin ${ }^{1,2, \#}$, Jean-Baptiste Jager ${ }^{2}$, Manon Tardif ${ }^{2}$, Emmanuel Picard ${ }^{2}$, Emmanuel Hadji ${ }^{2}$, Frédérique de Fornel $^{1}$, \\ and Benoît Cluzel ${ }^{1, *}$ \\ ${ }^{1}$ Groupe Optique de Champ Proche - L.R.C. SiNOPTIQ du C.E.A. nDSM-08-36, Laboratoire Interdisciplinaire Carnot de Bourgogne \\ U.M.R. C.N.R.S. 6303, Université de Bourgogne-Franche Comté, 9. Av. A. Savary, 21078 Dijon, France \\ ${ }^{2}$ Université Grenoble Alpes, C.E.A., I.N.A.C., PHELIQS, SINAPS, F-38000 Grenoble, France \\ \# Current address: RIES, Hokkaido University, Kita 20-jo Nishi 10-chome, Kita-ku, Sapporo, Hokkaido, 001-0020, Japan
}

\begin{abstract}
Due to the action of the scattering force, particles that are optically trapped at the surface of a waveguide are propelled in the direction of the light propagation. In this work, we demonstrate an original approach for creating tunable periodic arrays of optical traps along a few-mode silicon nanophotonic waveguide. We show how the near-field optical forces at the surface of the waveguide are periodically modulated when two guided modes with different propagation constants are simultaneously excited. The phenomenon is used to achieve stable trapping of a large number of dielectric particles or bacteria along a single waveguide. By controlling the light coupling conditions and the laser wavelength, we investigate several techniques for manipulating the trapped particles. Especially, we demonstrate that the period of the optical lattice can be finely tuned by adjusting the laser wavelength. This effect can be used to control the trap positions, and thus transport the trapped particles in both directions along the waveguide.
\end{abstract}

\section{Introduction}

Photonic waveguides are commonly used as on-chip optical conveyor belts in on-chip optical trapping and manipulation experiments. Kawata and Tani [1] first demonstrated in 1996 that particles in liquid solution can be trapped and propelled under the action of the optical force in the near-field of a photonic waveguide. Since then, various types of waveguides have been studied for optical trapping and transportation of particles: ridge [15], slot [6], photonic crystal [7], plasmonic [8], hollowcore waveguides [9], as well as micro- and nanofibres [10]. Waveguides have been mostly used for efficiently transporting micro- and even nanoparticles [6], as well as cells and bacteria in liquid solutions $[3,4,9]$. However, stable optical trapping of particles at a constant position along the waveguide is a more challenging task. In previous works, it required the use of a photonic or plasmonic resonator [11-20], or the presence of a standing wave along the waveguide, formed by two counter-propagating waves [21,22]. Some recent works suggested that the simultaneous excitation of two modes with different propagation constants could provide an alternative approach $[2,23,24]$.

In this work, we investigate the optical force distribution in the near-field of a few-mode silicon waveguide. Numerical simulation and optical trapping experiments are performed in order to study the periodic modulation of optical forces resulting from the simultaneous excitation of different pairs of guided modes [25]. Especially, we achieve creating near-field optical lattices that enable stable optical trapping of dielectric particles and bacteria. We also report on the use of such near-field optical lattices in order to handle in various ways the trapped particles [25].

\section{Results and discussion}

\subsection{Few-mode silicon waveguides}

Nanophotonic waveguides with rectangular crosssections were fabricated on a Silicon-On-Insulator chip using standard e-beam lithography process. The light from a tunable laser source emitting at telecom wavelengths was coupled into 5 - $\mu \mathrm{m}$-large waveguides using a lensed optical fibre. The position of the fibre relative to the waveguide entrance and the polarisation of the incident laser light were then used to control the excitation of the different guided modes in a few-mode nanophotonic waveguide on the chip.

In the case of a waveguide with a $510 \times 248 \mathrm{~nm}^{2}$ cross-section, three guided modes can be excited: two transverse-electric modes (TE0 and TE1) and one transverse-magnetic mode (TM0). When two guided modes are excited at the same time in the waveguide, the near-field optical forces are periodically modulated due to the difference of propagation constants. The period of the modulation follow the equation:

Corresponding author: benoit.cluzel $@$ u-bourgogne.fr 


$$
p_{\mathrm{ij}}=2 \pi /\left|\beta^{(\mathrm{i})}-\beta^{(\mathrm{j})}\right|=\lambda_{0} /\left|n_{\mathrm{eff}}^{(\mathrm{i})}-n_{\mathrm{eff}}^{(\mathrm{j})}\right|
$$

where $\lambda_{0}$ refers to the free-space wavelength and $\beta^{(\mathrm{i})}, \beta^{(\mathrm{j})}$ $\left(\right.$ respectively neff ${ }^{(i)}$, neff $^{(j)}$ ) to the propagation constants (respectively to the effective refractive indices) of the two guided modes. Because the propagation constant of each mode depends on the wavelength, we can evaluate the variation of the period of the optical force modulation based on numerical simulation results (Figure 1).

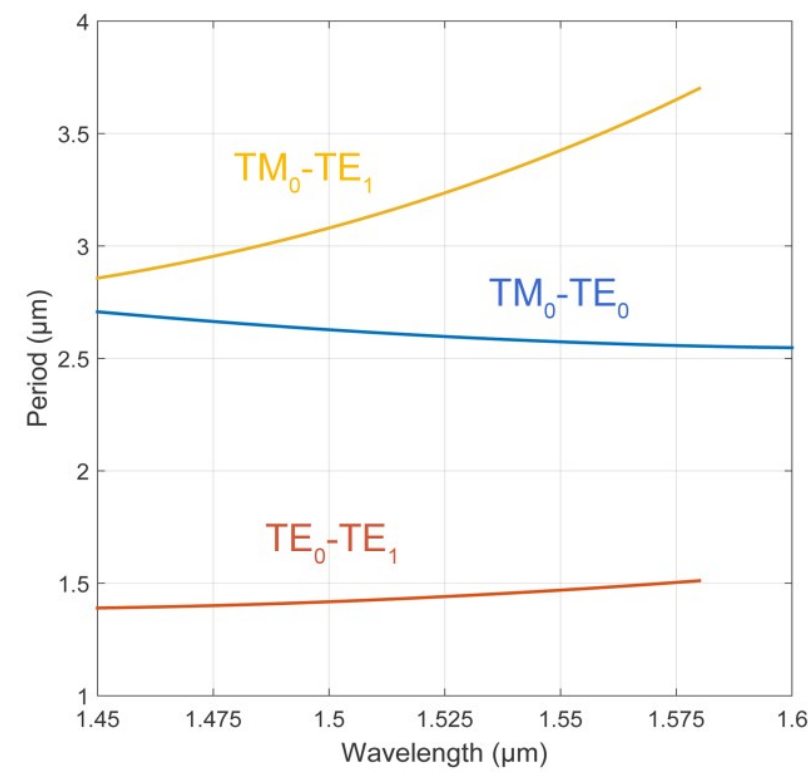

Fig. 1. Period of the optical force modulation as a function of the wavelength.

\subsection{Near-field optical lattices}

In some cases, the periodic modulation of the near-field optical forces can lead to the formation of a periodic array of optical traps along the surface of the waveguide. As shown in Figure 2, $1 \mu \mathrm{m}$ and $500 \mathrm{~nm}$ fluorescent polystyrene beads as well as e. coli bacterium cells were stably trapped in such optical lattices when both transverse-electric (TE) and transverse-magnetic (TM) modes were excited in the waveguide. In Figure 2(a), $500 \mathrm{~nm}$ beads were trapped in staggered rows on both sides of the waveguide with a period of $1.46 \mu \mathrm{m}$. In Figure 2(b), a periodic array of $1 \mu \mathrm{m}$ beads with a period of $3.26 \mu \mathrm{m}$ was formed above the waveguide. Those experimental results are in good agreement with numerical simulation results. Indeed, it was verified that the simultaneous excitation of both the fundamental TE and $\mathrm{TM}$ modes $\left(\mathrm{TE}_{0}\right.$ and $\mathrm{TM}_{0}$, respectively) is at the origin of the one-row periodic array of traps, while the simultaneous excitation of both the fundamental and the first order TE modes ( $\mathrm{TE}_{0}$ and $\mathrm{TE}_{1}$, respectively) is at the origin of the particles being trapped in staggered rows. (a)

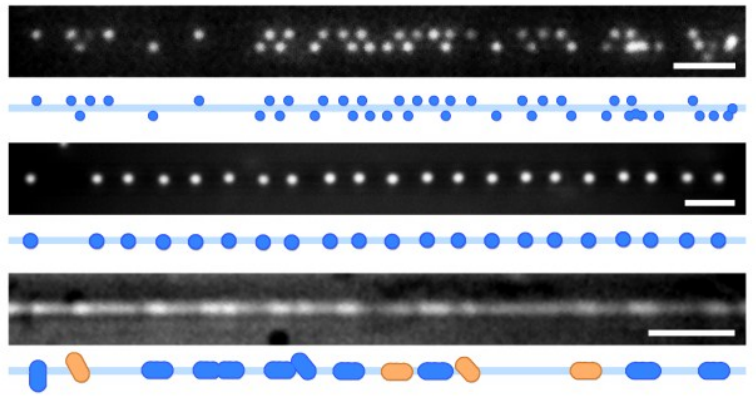

Fig. 2. Periodic arrangement of $500 \mathrm{~nm}$ polystyrene beads trapped in staggered rows. (b) Periodic arrangement of $1 \mu \mathrm{m}$ polystyrene beads above the waveguide. (c) Stable trapping of several e.coli bacteria above a single waveguide.

\subsection{On-chip optical manipulation}

The near-field optical lattices described above enable not only stable trapping of a large number of particles but also the development of new on-chip optical manipulation techniques. By modifying the polarization of the incident laser light or the light coupling conditions at the entrance of the photonic chip, it was possible to switch either from one optical lattice configuration to another, or from the stable trapping to the optical propulsion of the trapped particles. Moreover, it is possible to dynamically control the period of the optical lattice by tuning the laser wavelength. The particle spacing can therefore be finely tuned, and the position of the trapped particles precisely controlled along the waveguide. As shown in Figure 3, we were able to transport the trapped particles in both directions along the waveguide By continuously varying the laser wavelength: in direction of the light propagation when increasing the wavelength (forward transportation), and in the opposite direction when decreasing the wavelength (backward transportation).

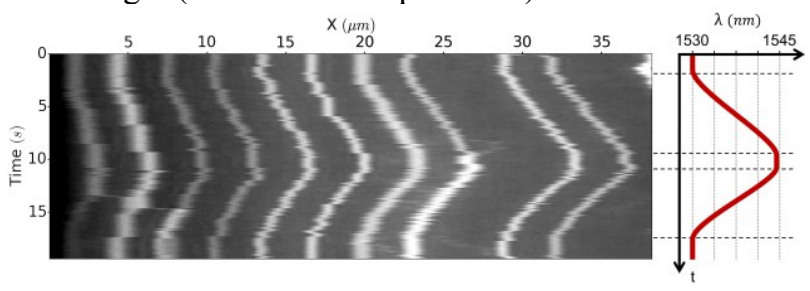

Fig. 3. Kymograph describing the continuous forward (to the right) and backward (to the left) transportation of $500 \mathrm{~nm}$ beads trapped in a $\mathrm{TM}_{0}-\mathrm{TE}_{1}$ lattice by continuously varying the wavelength.

\section{Conclusion}

In this work, it was demonstrated how a few-mode silicon waveguide can be used as a platform for creating one-dimensional near-field optical lattices in an optofluidic chip. It was shown that the simultaneous excitation of pairs of guided modes generates a periodic modulation of near-field optical forces along the waveguide. By selecting the adequate pairs of guided modes, we were able to stably trap a large number of particles and bacteria along a single waveguide. In 
addition, we showed how controlling the light coupling conditions and the laser wavelength makes it possible to finely tune the optical lattice properties. Taking advantage of the lattice tunability, we demonstrated several on-chip optical manipulation techniques for handling the trapped particles. The optical trapping and manipulation methods demonstrated in this work can be readily implemented in lab-on-a-chip devices and should pave the way for various optofluidic applications.

This work was performed in cooperation with the Labex ACTION program (contract ANR-11-LABX-0001-01), and with the support of the "Plateforme Technologique Amont" of Grenoble, funded through the CNRS Renatech network.

\section{References}

1. S. Kawata, T. Tani, Opt. Lett. 21, 1768 (1996)

2. T. Tanaka, S. Yamamoto, Jpn. J. Appl. Phys. 41, L260 (2002)

3. S. Gaugiran, S. Gétin, J.M. Fedeli, G. Colas, A. Fuchs, F. Chatelain, J. Dérouard, Opt. Express 13, $6956(2005)$

4. B.S. Ahluwalia, P. McCourt, T. Huser, O.G. Hellesø, Opt. Express 18, 21053 (2010)

5. P. Schein, D. O'Dell, D. Erickson, ACS Photon. 4, 106 (2017)

6. A.H. Yang, S.D. Moore, B.S. Schmidt, M. Klug, M. Lipson, D. Erickson, Nature 457, 71 (2009)

7. M.G. Scullion, Y. Arita, T.F. Krauss, K. Dholakia, Optica 2, 816 (2015)

8. C. Yang, D. Pan, L. Tong, H. Xu, Nanoscale 8, 19195 (2016)

9. S. Unterkofler, M.K. Garbos, T.G. Euser, P.S.J. Russell, J. Biophotonics 6, 743 (2013)

10. G. Brambilla, G.S. Murugan, J.S. Wilkinson, D.J. Richardson, Opt. Lett. 32, 3041 (2007)

11. S. Mandal, X. Serey, D. Erickson, Nano Lett. 10, 99 (2009)

12. C. Renaut, J. Dellinger, B. Cluzel, T. Honegger, D. Peyrade, E. Picard, F. de Fornel, E. Hadji, Appl. Phys. Lett. 100, 101103 (2012)

13. C. Renaut, B. Cluzel, J. Dellinger, L. Lalouat, E. Picard, D. Peyrade, E. Hadji, F. de Fornel, Sci. Rep. 3, 2290 (2013)

14. S. Lin, K.B. Crozier, ACS Nano 7, 1725 (2013)

15. C. Pin, B. Cluzel, C. Renaut, D. Peyrade, E. Picard, E. Hadji, F. de Fornel, Appl. Phys. Lett. 105, 171108 (2014)

16. C. Pin, B. Cluzel, C. Renaut, E. Picard, D. Peyrade, E. Hadji, F. de Fornel, ACS Photon. 2, 1410 (2015)

17. C. Pin, C. Renaut, M. Tardif, J.-B. Jager, E. Delamadeleine, E. Picard, D. Peyrade, E. Hadji, F. de Fornel, B. Cluzel, Proc. SPIE 10252, 1025212 (2017)

18. P.T. Lin, H.Y. Chu, T.W. Lu, P.T. Lee, Lab. Chip 14, 4647 (2014)
19. G. Magno, A. Ecarnot, C. Pin, V. Yam, P. Gogol, R. Mégy, B. Cluzel, B. Dagens, Opt. Lett. 41, 3679 (2016)

20. A. Ecarnot, G. Magno, V. Yam, B. Dagens, Opt. Lett. 43, 455 (2018)

21. M. Soltani, J. Lin, R.A. Forties, J.T. Inman, S.N. Saraf, R.M. Fulbright, M. Lipson, M.D. Wang, Nature Nanotech. 9, 448 (2014)

22. J.E. Baker, R.P. Badman, M.D. Wang, WIREs Nanomed. Nanobiotechnol. 10, e1477 (2018)

23. O.A. Schmidt, T.G. Euser, P.S.J. Russell, Opt. Express 21, 29383 (2013)

24. T.H. Stievater, D.A. Kozak, M.W. Pruessner, R. Mahon, D. Park, W.S. Rabinovich, F.K. Fatemi, Opt. Mat. Express 6, 3826 (2016)

25. C. Pin, J.-B. Jager, M. Tardif, E. Picard, E. Hadji, F. de Fornel, B. Cluzel, Lab. Chip 18, 1750 (2018) 